\title{
" De la diversité des parcours et des formations dans les collèges du Bas-Canada : le cas de Montréal (1789-1860) ${ }^{1}$ "
}

\section{Ollivier Hubert}

\begin{abstract}
RÉSUMÉ
Faisant usage de registres d'inscriptions et de registres de compte d'élèves, cette étude produit un portrait de la population d'un collège catholique montréalais entre 1789 et 1860 . Il s'en dégage une grande hétérogénéité, particulièrement dans les cheminements. On retrouve ainsi une situation qui ressemble à celle des institutions françaises et provinciales d'Ancien Régime. En fait, le Collège de Montréal apparaît comme une maison d'éducation polyvalente, ouverte à des clientèles diversifiées et capable de leur offrir des formations adaptées à la pluralité des attentes et des moyens. Au total, l'image du pensionnaire engagé dans de longues études poursuivies à l'ombre des murs d'austères séminaires semble largement à réviser.
\end{abstract}

\section{ABSTRACT}

Based on the registration and account records of students, this study provides a portrait of the population of a Catholic college in Montreal between 1789 and 1860. A picture of strong heterogeneity thus emerges, particularly in the orientations. A situation resembling that of the French provincial institutions from the Old Regime is thus found. In fact, the College of Montreal is seen to be home to multi-purposed education, open to a diversified clientele and capable of offering education adapted to multiple expectations and abilities. In all, the image of a boarder engaged in long study periods pursued in the shadows of the walls of austere seminaries appears to need major revision.

Il existe à propos des collèges " classiques " québécois quelques opinions fort répandues mais bien peu fondées. Parmi celles-ci, l'idée selon laquelle ces écoles d'initiation au modèle humaniste auraient été élitistes par définition, parfaitement efficaces à produire une caste cléricale et, accessoirement, à reproduire la petite bourgeoisie canadienne-française. Or, s'il est incontestable que seule une minorité des garçons de chaque classe d'âge arpenta les couloirs des collèges, rien, ou presque, ne nous permet de qualifier cette minorité 2 . La sociologie collégienne reste largement une inconnue. Nous ne savons des élèves ni le milieu social d'origine ni celui d'arrivée. Nous ne possédons aucune mesure précise de l'investissement que les études représentaient 
pour les familles. Nous ne connaissons par voie de conséquence rien, ou à peu près, des attentes des acteurs concernés, des appartenances diverses des enfants et jeunes gens qui se côtoyaient dans les mêmes salles et donc de la nature de la microsociété qui s'y constituait. Au bout du compte, nous avons encore beaucoup à apprendre sur le degré et les modalités de la pénétration de la culture classique dans la société québécoise du XIX siècle.

La minceur des informations disponibles n'a pourtant pas empêché un « discours " (au sens foucaldien du $\operatorname{mot}^{3}$ ), de fonctionner et de proposer l'image d'un collège élitiste et clérical, véritable stéréotype dont il conviendrait du reste de produire la généalogie $e^{4}$. Face à ce double constat — insuffisance de renseignements fondés à propos de la nature et de l'évolution des populations collégiales et caractère pléthorique des textes qui prétendent livrer sur elles une vérité-la recherche est orientée dans deux directions qui devraient permettre de définir une histoire sociale des collèges classiques. La première consistera à déconstruire avec minutie les logiques du discours afin d'en dévoiler les intentions, de faire voir ses divers supports et auteurs et finalement d'en déterminer la fonction dans les luttes sociales. La seconde cherchera à produire un portrait aussi détaillé que possible de la fréquentation effective du secteur dit " secondaire ", et à terme de l'ensemble des éléments qui en font l'histoire concrète. De l'écart ainsi signalé entre l'usage des établissements et leurs représentations pourra émerger une compréhension du collège comme institution socialement et historiquement constituée par un appareil complexe de facteurs interdépendants.

Nous amorçons cette enquête en tâchant de déterminer, dans un cas spécifique et pour une période délimitée, qui étaient les élèves et surtout quels étaient les différents types de parcours envisageables pour eux. Faisant retour à une approche quantitative boudée par la production historienne des dernières décennies, cet article veut contribuer à préciser les caractéristiques des cohortes du XIX ${ }^{\mathrm{e}}$ siècle. C'est moins, à la manière des études de l'histoire sociale classique, à la répartition des collégiens en fonction du métier exercé par le père que nous nous attacherons qu'à la reconstitution des itinéraires. Cette identification permettra de révéler la diversité des clientèles, la pluralité des formations et la variété des cheminements, aspects que le discours oblitérait largement. Elle signale au total le caractère plastique des collèges en tant que caractéristique dominante de leur histoire; une plasticité qui répondant aux demandes de scolarisation changeantes et multiples issues du milieu, nous éloigne de l'image de raideur traditionaliste projetée et par les laudateurs et par les éreinteurs du système.

\section{A-À la croisée de deux modèles}

L'historiographie des populations collégiales propose des résultats contrastés. Lorsqu'elle concerne des temps anciens $\left(\mathrm{XVI}^{\mathrm{e}}, \mathrm{XVII}^{\mathrm{e}}\right.$ et $\mathrm{XVIII}{ }^{\mathrm{e}}$ siècles) et travaille dans une perspective chronographique, elle décrit globalement un état de mixité sociale $^{5}$. Loin d'être un bastion élitaire, le collège est alors le lieu où se rencontrent nobles, notables et élites populaires. Si elle prend au contraire pour point de départ le milieu du $\mathrm{XX}^{\mathrm{e}}$ siècle, moment de la critique radicale du vieux modèle humaniste, et remonte en amont en une démarche généalogique, l'analyse de la fréquentation 
des établissements secondaires a plutôt tendance à les établir en tant que sites privilégiés de la domination bourgeoise ${ }^{6}$. Les deux représentations ne sont pas forcément contradictoires $^{7}$, mais dessinent un vrai problème d'histoire, qui est de caractériser socialement l'évolution de ce type de maisons d'enseignement au XIX siècle. Claude Galarneau a fait paraître, en 1974, une étude intéressante à cet égard parce que, considérant une très longue période (1620-1970), elle s’offre la possibilité de décrire une modification susceptible de rendre intelligible l'existence des deux visions historiographiques susmentionnées. Fruit de la rencontre de l'école érudite et de l'histoire sociale, l'ouvrage présente un panorama remarquable, mais ne parvient pas à dresser un portrait cohérent de la transformation des clientèles ${ }^{8}$. Il semble conclure à une dichotomie fondamentale entre les collèges ruraux, plus démocratiques dans leur recrutement et produisant essentiellement des prêtres, et les collèges urbains, plus élitistes et orientés vers les professions libérales. Galarneau souligne cependant la connaissance "sommaire " qu'il a de ces questions, en particulier pour ce qui est des établissements citadins (Québec et Montréal) parce que leurs archives sont, selon son appréciation, " pauvres en renseignements pour ce qui concerne l'inscription des élèves" ".

Celles du Collège de Montréal possèdent pourtant une série presque complète de registres de comptes d'élèves et de registres d'inscription qui permettent une reconstitution assez précise de la population de cette institution depuis $1789^{10}$. Certes, la profession du père n'est jamais indiquée, et le nom même de celui-ci n'apparâ̂t pas avant les années 1850, ce qui rend difficile dans l'immédiat une analyse de type strictement sociographique ${ }^{11}$. Cependant, à partir des données disponibles (âge, statut, provenance géographique, classe fréquentée, parcours scolaire), on peut tracer les contours d'une histoire sociale de cette maison. Elles permettent en effet d'établir non seulement un portrait détaillé de la population collégienne, mais encore de mieux saisir les évolutions de la structure qui les accueille. Cette enquête montrera un état initial d'hétérogénéité et une progressive homogénéisation. École des élites religieuses, professionnelles et marchandes au XVIII ${ }^{e}$ siècle, mais surtout des petits commerçants, artisans et même de garçons issus du monde populaire, le Collège de Montréal devient graduellement, au long du XIX ${ }^{\mathrm{e}}$ siècle, le site de production et de reproduction des seules catégories bourgeoises.

\section{B-La croissance du nombre de pensionnaires}

La mise en série des effectifs annuels permet de dessiner la courbe du recrutement (Graphique 1).

Les quelques chiffres obtenus pour la dernière décennie du XVIII ${ }^{e}$ siècle révèlent que le Collège, qui se nomme alors Collège Saint-Raphäl et n'est que partiellement sulpicien, est un établissement d'une centaine d'élèves, ce qui en fait une maison de taille modeste, même pour l'époque. L'édifice brûle en 1803 et Saint-Sulpice finance pour le remplacer un " petit séminaire " dont la capacité est nettement plus importante. Une vingtaine d'années après sa fondation, la nouvelle entité atteint un sommet de recrutement en accueillant 340 collégiens. Par la suite, la moyenne annuelle oscille plutôt autour de 200 inscrits, malgré une pointe au milieu des années 1840 . 


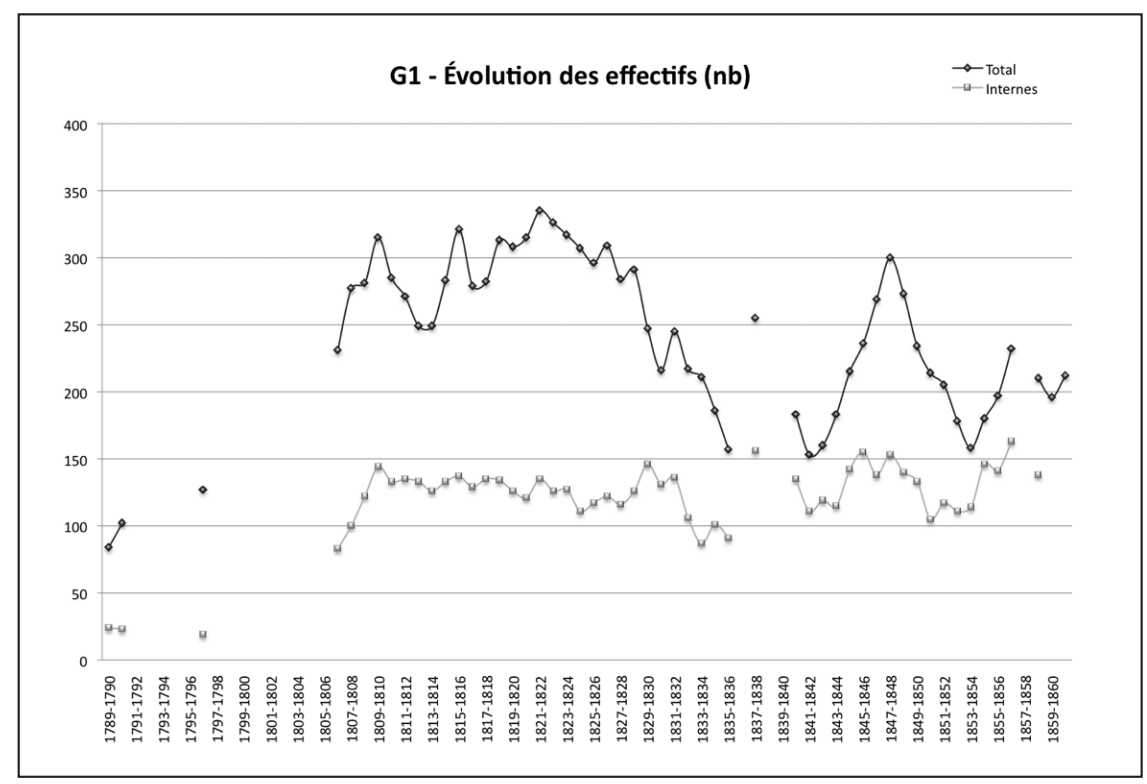

Les observateurs historiens des statistiques collégiales connaissent bien ces fluctuations qui sont le produit de causes conjuguées : difficultés économiques des parents, incertitudes politiques, épidémies, concurrences des institutions éducatives. Dans le cas qui nous intéresse, toutes ces raisons peuvent être évoquées. Le prix de la pension augmente sensiblement en 1832 et celui de la scolarité en 1840. Les crises constitutionnelles des années 1830 peuvent avoir joué un rôle d'autant plus important que les sulpiciens prennent parti. Des collèges anglo-protestants, puis une maison jésuite bilingue ouvrent à Montréal respectivement en 1822, 1842 et 1848. Enfin, il existe des fluctuations dans la demande sociale pour l'étude des langues anciennes, bien documentée dans le cas de la France ${ }^{12}$, mais qui reste à établir pour le Québec.

Un facteur met sur la piste de ce qui semble être l'explication fondamentale pour rendre compte des évolutions observées dans le nombre des élèves accueillis. On réalise en effet que, si les effectifs globaux connaissent d'importantes variations, celui des internes est remarquablement stable (entre 100 et 150 garçons). Ce sont donc les très spectaculaires écarts dans le chiffre des externes qui expliquent presque à eux seuls le dessin de la courbe générale. Or, la distinction entre pensionnaires et externes possède une dimension sociale évidente, puisque le prix de la pension est assez élevé pour rendre le statut d'interne inaccessible aux petites classes urbaines. Les ondulations des effectifs sont par conséquent révélatrices du degré d'ouverture du Collège à l'égard des familles les moins favorisées. Comme la population collégiale totale enregistre globalement une baisse entre 1820 et 1860 et que, par ailleurs, le nombre de pensionnaires reste stable, il faut conclure que l'établissement, qui était un modeste externat à la fin du XVIII ${ }^{e}$ siècle, est progressivement devenu un pensionnat durant la première moitié du XIX siècle (Graphique 2).

Ce phénomène est tout à fait fondamental et inscrit le Collège de Montréal 


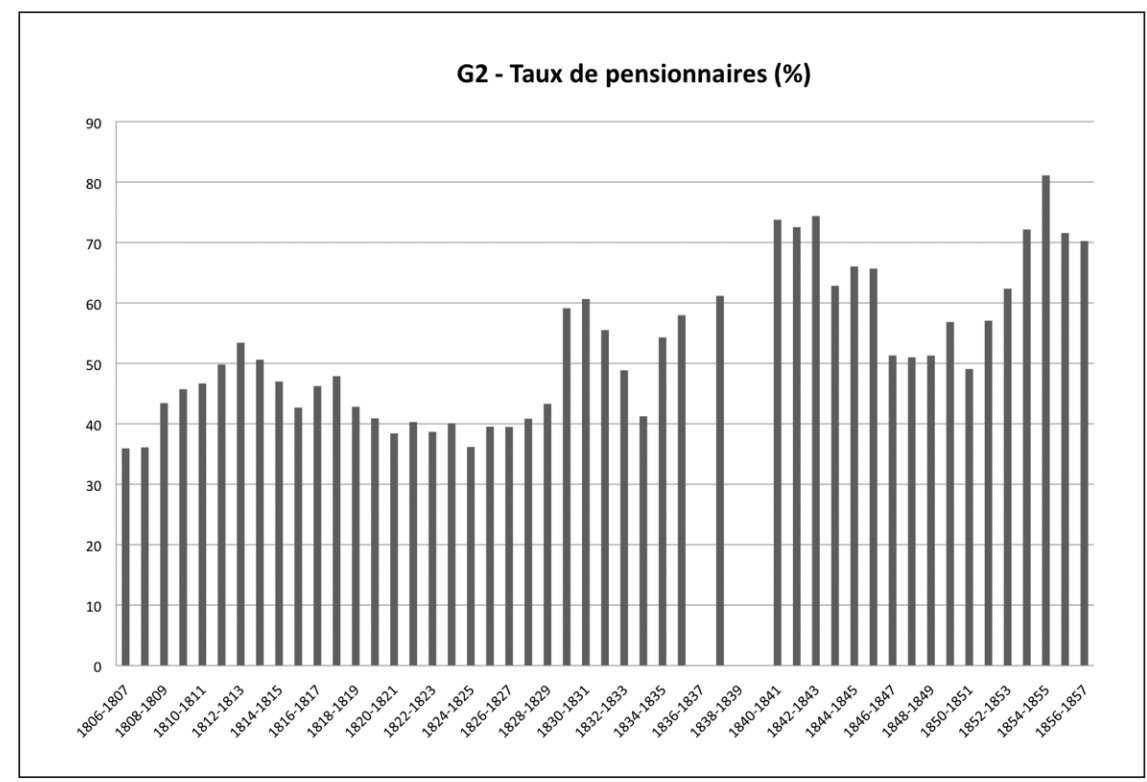

dans une tendance internationale qui voit les classes aisées graduellement adhérer à l'idée qu'un bon gouvernement des jeunesses masculines implique une séparation d'avec le milieu familial ${ }^{13}$. Le collège se transforme alors, pour répondre aux attentes neuves de la bourgeoisie montante, qui le considère non plus seulement comme un lieu de scolarisation, mais comme un dispositif éducatif au sens large. Comme il s'agit d'un luxe, l'institution doit pour survivre offrir un service qui rejoint les désirs de la clientèle. Ces exigences sont en partie matérielles, ce qui explique que l'établissement déménage en 1860 dans des locaux modernes situés dans un secteur bucolique. Elles relèvent aussi du préjugé de classe : les parents espèrent que leur rejeton fréquentera des enfants du même milieu que lui, ou mieux encore d'un milieu supérieur au sien, camarades qui seront pour lui autant d'exemples à suivre et constituer des relations sociales utiles et profitables dans l'avenir. Comme le résume Madeleine Compère : "si l'externat peut tolérer une large ouverture sociale, le pensionnat tend à l'homogénéité ${ }^{14} »$.

\section{C_L’origine géographique des élèves}

La provenance géographique des élèves constitue le second paramètre classique de l'analyse des populations collégiales. Cette donnée est généralement utilisée dans une logique d'évaluation de la performance de l'offre éducative. Du reste, étonnamment, on se rend compte qu'un large comme un étroit bassin de recrutement peuvent être considérés de manière également positive : dans le premier cas, on dira qu'il témoigne $\mathrm{du}$ " rayonnement " d'une institution, dans le second qu'il signale un bon maillage du territoire par réseau d'établissements secondaires ${ }^{15}$. Les chiffres obtenus pour le Collège de Montréal à cet égard corroborent globalement ceux déjà livrés par les 
quelques études qui avaient tenté l'exercice pour d'autres maisons d'enseignement du même type au Québec ${ }^{16}$. La provenance est locale d'abord, c'est-à-dire montréalaise puis régionale (Graphique 3).

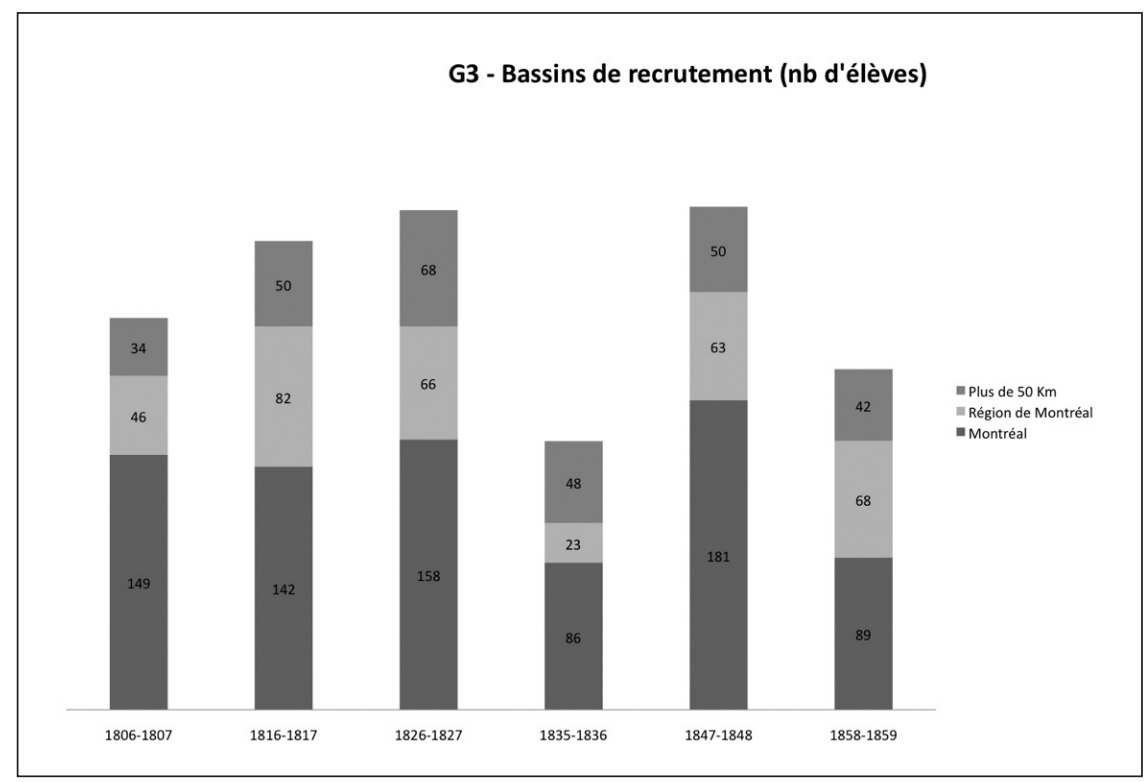

Durant la première moitié du XIX siècle, la part prise par chaque bassin est relativement stable. Les Montréalais ont toujours occupé entre 50 et $60 \%$ des places, les enfants domiciliés à l'intérieur d'une couronne de $50 \mathrm{~km}$ autour de la métropole entre 20 et $30 \%$ et ceux qui venaient de plus loin entre 15 et $20 \%$ (sauf pendant le creux atypique du milieu des années 1830). Dans les années 1850 toutefois, on constate une baisse majeure des inscriptions montréalaises (Graphique 4) et une affirmation de la présence des collégiens issus de la région métropolitaine.

Faut-il relier étroitement la fluctuation du taux de pensionnaires avec celle du taux de candidats provenant de l'extérieur de Montréal? Autrement dit les Montréalais sont-ils les externes et les non-Montréalais (ou " forains ») les internes? En fait, les choses sont plus nuancées puisqu'une proportion importante de citadins sont internes (entre 15 et $40 \%$ ) et que sensiblement la même proportion des "forains " sont externes. Deux réalités, minoritaires, mais significatives, sont par conséquent à souligner : l'existence de jeunes vivant une vie de pensionnaire à deux pas du domicile familial et celles de collégiens placés loin de leurs familles, mais à l'extérieur de l'établissement sulpicien, chez un logeur ou un membre de la parenté. Le pensionnat n'est donc ni entièrement une question purement logistique, ni un choix purement éducatif ou stratégique. L'évolution que marquent les courbes présentées pousse cependant à mettre de l'avant le facteur de distinction sociale. La pension coûte 360 livres à la fin du XVIII e siècle, 500 livres au XIX siècle, somme à laquelle s'ajoutent les frais de fournitures et de déplacement. Les sulpiciens offrent des bourses, mais 


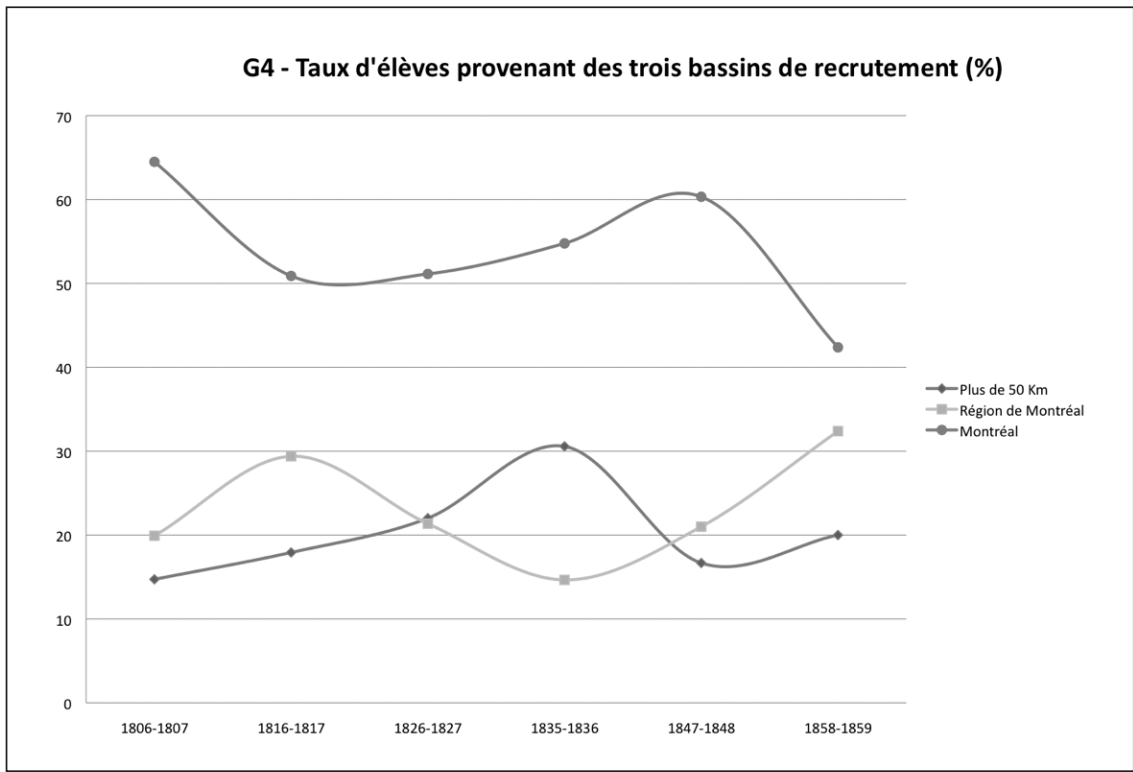

elles ne concernent que quelques enfants pauvres qui promettent de devenir prêtres. En somme, le pensionnat est inaccessible aux couches moyennes de la société, particulièrement aux fils de paysans, commerçants ou artisans. Les citadins aisés, qui n’en ont aucune nécessité, sont pourtant de plus en plus nombreux à choisir la formule de l'internat (Graphique 5A).

De même, si les forains de plus modeste origine pouvaient encore, jusque dans les

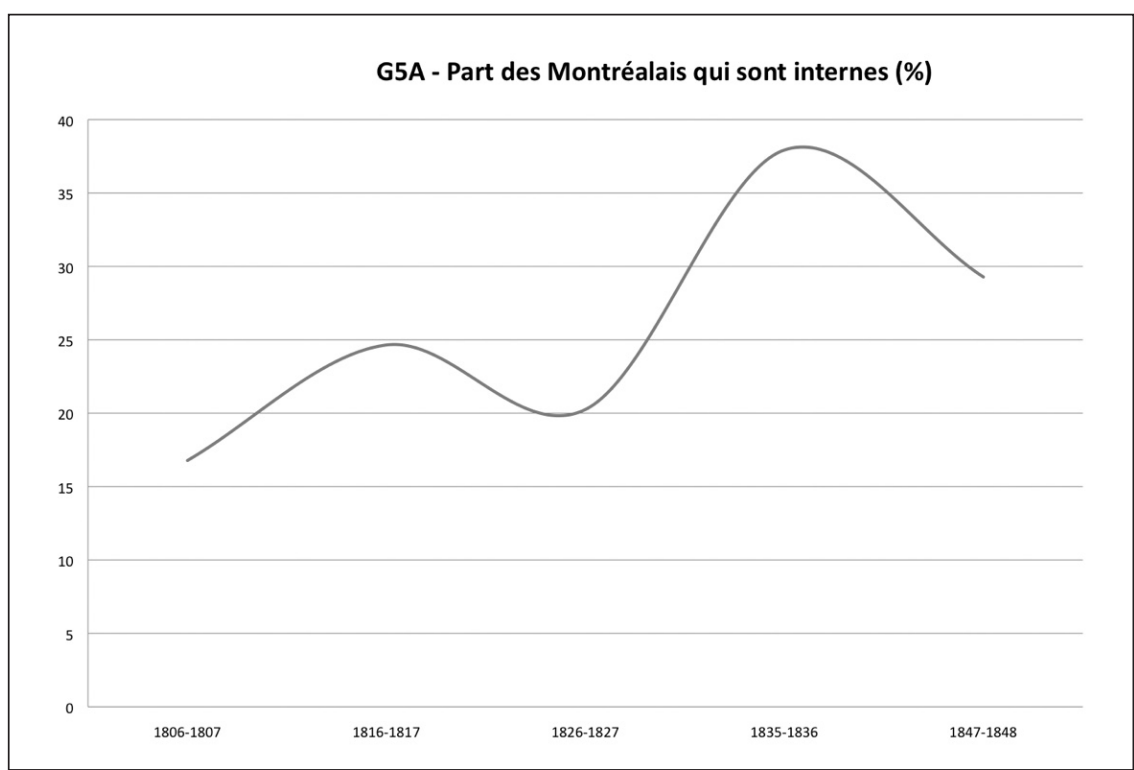




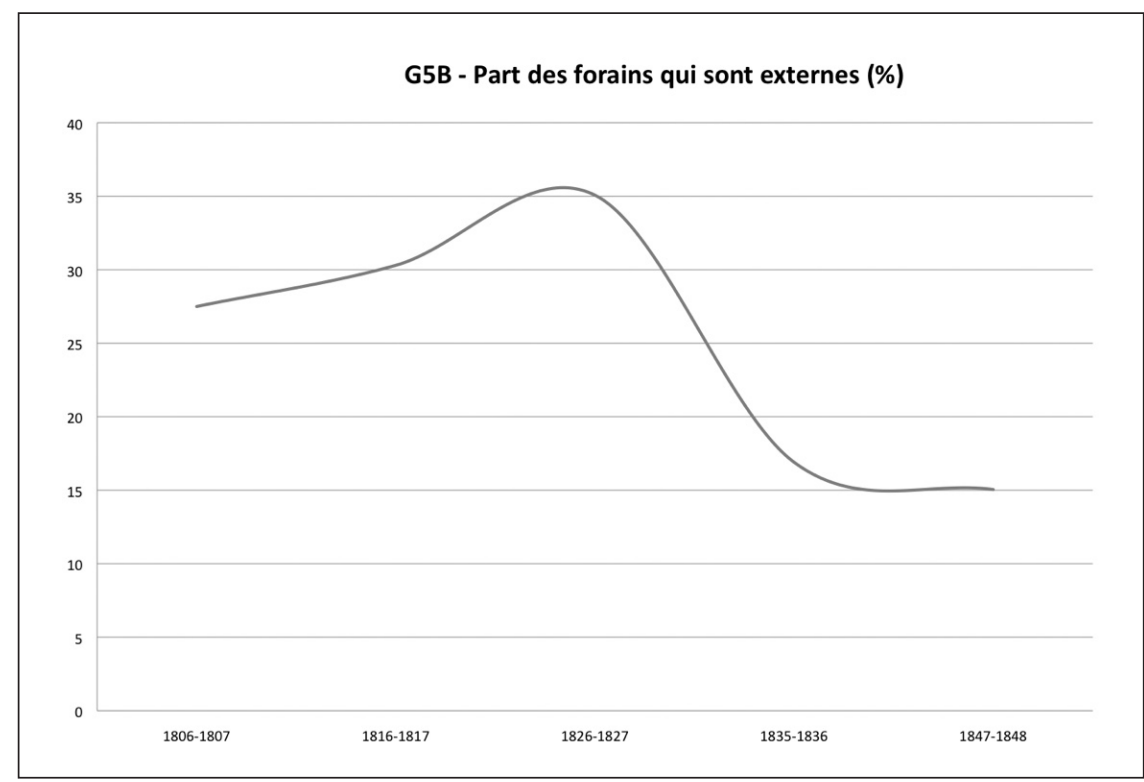

années 1820, prendre pension à l'extérieur du collège, cette solution moins onéreuse semble disparaître dans les années 1830 (Graphique 5B).

Le pensionnat s'impose donc comme système dominant au cours de notre période, très précisément à la rentrée de 1829 tandis que pour la première fois dans l'histoire du Collège, le nombre de pensionnaires dépasse celui des externes. Cette évolution possède à l'évidence une dimension stratifiante puisque le coût de la pension en réserve l'accès aux milieux les plus fortunés. L'identité du Collège de Montréal bascule alors progressivement vers le modèle du pensionnat bourgeois.

Même si leur importance numérique est assez faible, la curiosité pousse à en savoir plus sur le groupe de ceux dont le domicile familial est situé à plus de $50 \mathrm{~km} \mathrm{du}$ Collège (Graphique 6).

Dans les trois premières décennies du XIX e siècle, il s'agit surtout d'enfants issus de la colonie même, ce qui reflète tout simplement le sous-développement du réseau éducatif québécois à cette période ${ }^{17}$. À partir des années 1830 cependant, un nombre croissant d'Américains, provenant des États limitrophes, fréquentent le Collège de Montréal : ils forment jusqu'à $10 \%$ des inscrits en 1858 . Contrairement à l'hypothèse mise de l'avant par Claude Galarneau ${ }^{18}$, leurs patronymes suggèrent qu'ils ne sont pas Franco, mais Anglo-Américains. Il existe manifestement au sud de la frontière une clientèle fortunée, peut-être catholique, et que les prêtres cultivent. Leurs archives contiennent en effet les traces de publicités diffusées dans des quotidiens états-uniens ${ }^{19}$. Ces Américains attirent l'attention des chercheurs par leur exotisme ${ }^{20}$, mais leur altérité linguistique est loin d'être radicale dans le contexte de collèges aux populations diversifiées. Ainsi, entre le quart et le tiers des élèves du Collège des sulpiciens possèdent un nom à consonance britannique (Graphique 7).

Au-delà des fluctuations observées dans leur importance relative au sein de la 
G6 - Origine géographique des collégiens qui viennent de plus de $50 \mathrm{Km}$ (nb)
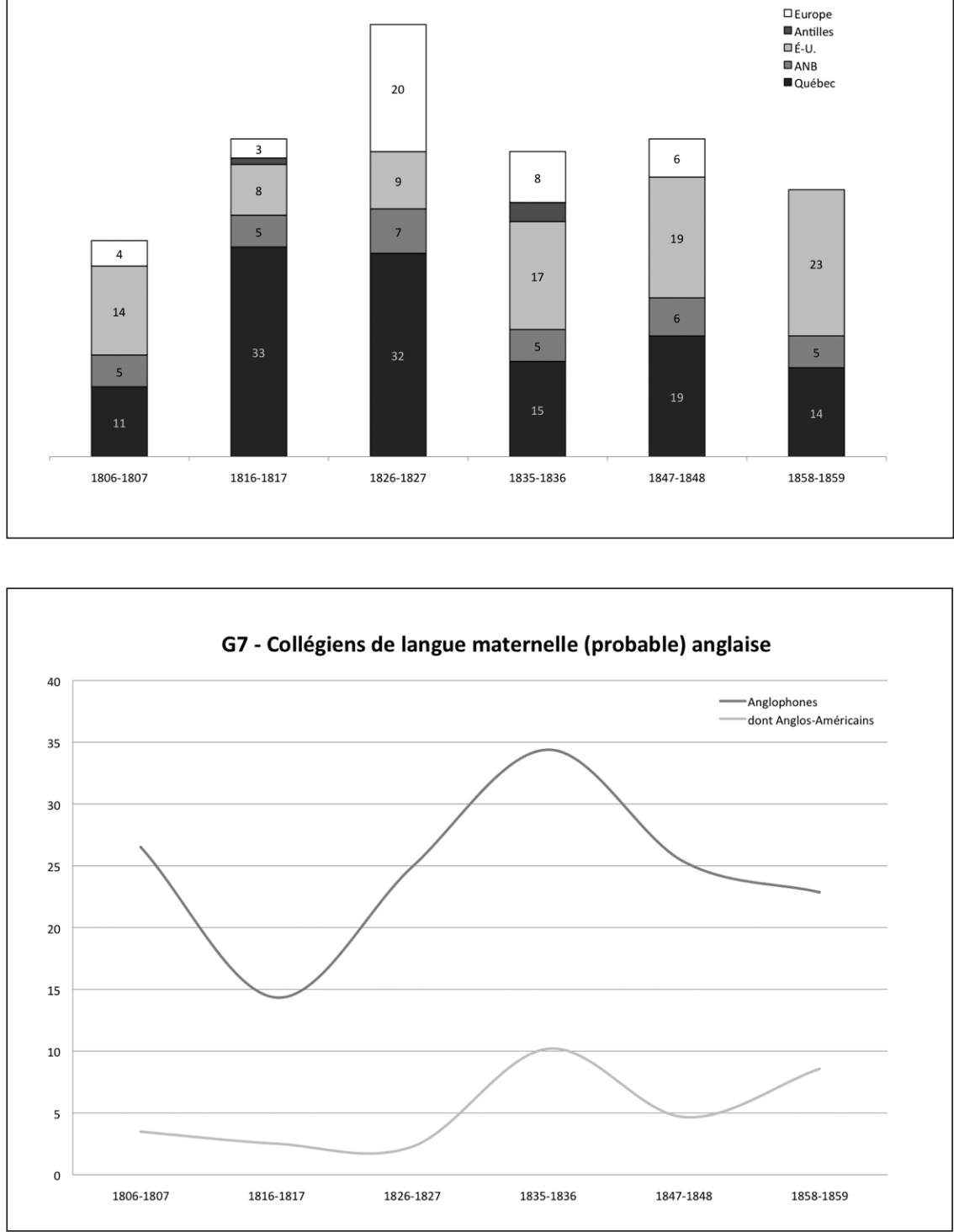

maison, c'est la présence d'une forte minorité d'Anglo-Montréalais qui doit ici être relevée, car elle exprime une caractéristique assez peu étudiée par l'historiographie québécoise de l'enseignement secondaire. Un collège, à Montréal, au XIX ${ }^{\mathrm{e}}$ siècle, apparaît comme une institution plurilingue (grec, latin, français, anglais). Se manifeste une évidente demande pour l'apprentissage des langues; un phénomène qui, à 
ma connaissance, n'a pas fait l'objet de recherche ${ }^{21}$. Notons aussi à ce chapitre que, si l'établissement traverse un processus d'homogénéisation, elle est, avant 1860 tout au moins, sociale plutôt linguistique puisque la part des anglophones reste constante.

Les données jusqu'ici analysées sont classiques. Elles permettent de situer le Collège de Montréal à l'intérieur d'un ensemble, celui des maisons d'enseignement secondaire de son époque. Si l'on s'en tient aux grands paramètres évoqués (nombre total d'élèves, pourcentage de pensionnaires et provenance géographique), il apparaît comme une institution de taille moyenne, desservant une population essentiellement locale et de plus en plus régionale, offrant un service de pensionnat pour la portion la plus fortunée de sa clientèle. Clientèle et service qui prennent avec le temps une importance manifeste, de sorte que c'est l'identité de l'établissement qui s'en trouve peu à peu transformée. Est-il possible, avec les données dont nous disposons, d'aller plus loin? Une proposition annoncée il y a quelques années par le Service d'histoire de l'éducation, important centre de recherche français, suggère que des connaissances nouvelles pourront être produites en pénétrant plus avant dans les "logiques d'établissement ${ }^{22}$ ». C'est dans l'esprit de cet effort inductif que je tente dans les lignes qui suivent de dépasser les analyses classiques pour entrer «à l'intérieur » du Collège et de relire les champs jusqu'ici considérés à la lumière d'un autre, plus rarement exploité : l'évolution de la structure pédagogique propre à l'établissement.

\section{D-Le portrait revisité de la population collégiale en tenant compte des " écoles " et des « classes "}

La plupart des études qui se penchent sur la fréquentation scolaire soulignent deux faits massifs qui surprennent, car ils cadrent mal avec l'idée que, de nos jours, on se fait d'une école secondaire. D'une part, l'on trouve parmi les inscrits une grande variabilité dans les âges : de très jeunes candidats et d'autres très âgés. D'autre part, seule une infime minorité des garçons achève le cours complet. Le premier phénomène, lorsqu'il est relevé, est généralement attribué à l'existence d'une formation préparatoire qui précède le cursus latin proprement dit ${ }^{23}$. Ce cours cependant est négligé par l'historiographie québécoise qui y voit une annexe négligeable à l'activité principale d'un collège, perçue comme étant la production de "finissants ". Le second phénomène est souvent présenté comme la preuve des défauts fondamentaux du système, qui sont mis de l'avant afin de justifier les changements apportés au milieu du XX $\mathrm{XX}^{\mathrm{e}}$ siècle. Les collégiens seraient poussés hors de l'établissement pour des causes variées : manque de moyens des parents, manque de courage ou d'aptitudes intellectuelles des élèves, sévérité excessive des institutions ou, au contraire, manque de sélection à l'entrée. Tout cela relève à mon sens d'un tropisme historiographique qui consiste à appréhender le collège en fonction d'un collégien idéal forgé à la fin du $\mathrm{XIX}^{\mathrm{e}}$ siècle : le pensionnaire qui subit une formation latine complète. Or, cet élève théorique et le type de dispositif pédagogique qu'il incarne ne correspondent pas à l'expérience éducative collégiale réelle de l'écrasante majorité, tout au moins pour la période considérée.

Le Collège de Montréal n'échappe pas aux prétendus défauts de système identifiés 
par l'historiographie. L'amplitude des âges est remarquablement étendue ${ }^{24}$. En 1826 par exemple, le petit George F. Bigelow, élève à l'école française, n'a que 5 ans. À l'autre extrémité de la pyramide, le philosophe Antoine Perrier est un adulte de 24 ans. Cette plasticité indique clairement le caractère plurifonctionnel de l'établissement et invite à se déprendre des perspectives contemporaines qui font $\mathrm{du}$ "secondaire " un ordre possédant une place précise dans un parcours intégré : entre le primaire et le supérieur. Suivant en cela le modèle commun aux sociétés européennes ou de culture européenne du XIX siècle, le Collège de Montréal n'a pas en charge une étape de la formation scolaire, mais un groupe d'étudiants. Plutôt qu'un niveau d'enseignement (du second degré), il se présente comme un environnement éducatif. Ignorer cette dimension pousse à survaloriser l'étude d'apprentissages qui préparaient au supérieur et ne concernaient qu'une fraction des élèves, comme la philosophie ou la rhétorique ${ }^{25}$. Or, le collège fonctionnait avec une autre logique, qui était de satisfaire les besoins multiples d'une clientèle diversifiée. Cette cohérence propre considérée, la diversité des âges semble moins fantastique et le taux de départs en cours de cheminement beaucoup moins dramatique.

Les registres de l'économe permettent de connaître la classe ou le cours fréquentés par l'enfant. Une répartition graphique des données compilées (Graphique 8) fait immédiatement apparaître une structure éducative qui ne correspond nullement à la succession régulière des classes s'organisant comme autant de niveaux à travers lesquels le jeune doit nécessairement cheminer ${ }^{26}$.

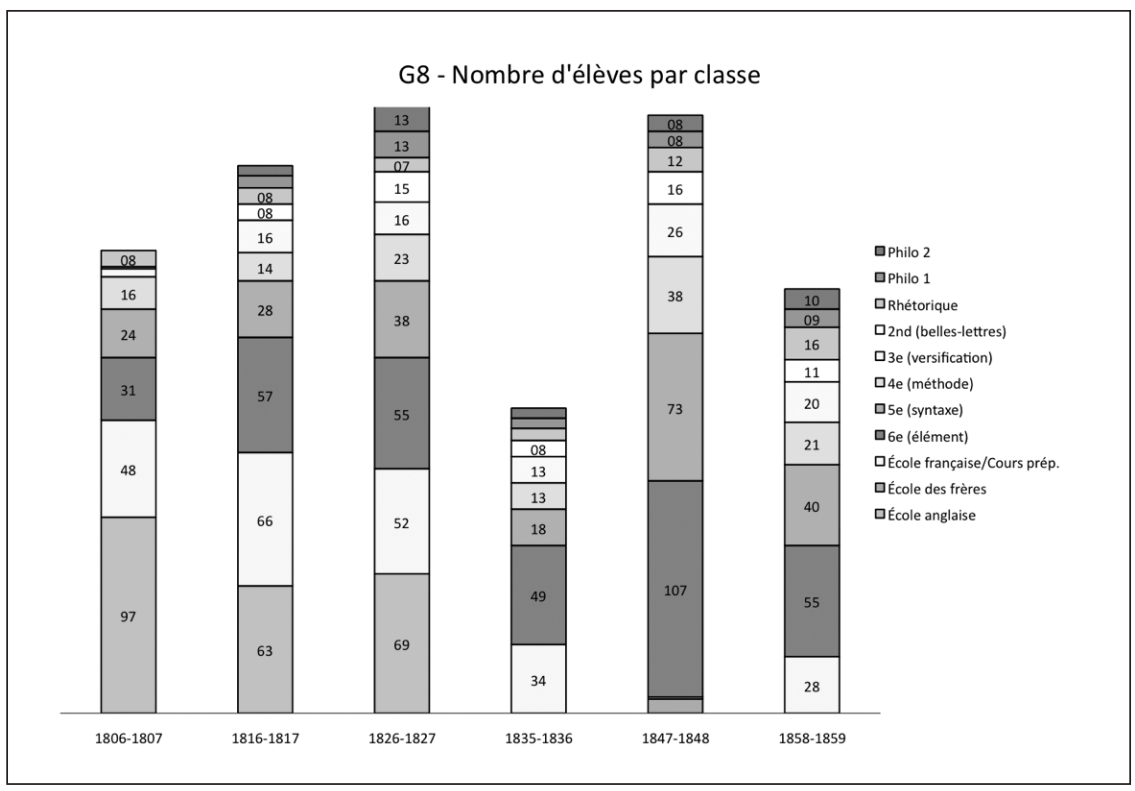

Les cahiers d'inscription montrent aussi nettement que les responsables du Collège comprennent leur institution comme offrant trois types de formation selon la langue principale d'enseignement. Ainsi, le directeur, lorsqu'il doit indiquer vers où l'enfant 
doit être aiguillé, inscrit-il, en fonction des désirs des parents et de sa propre évaluation du candidat : "pour l'anglais », " pour le français » ou " pour le latin ». Il y a donc trois filières distinctes vers lesquelles les nouveaux sont chaque année orientés. Elles sont parfois désignées dans les documents de la fin du XVIII ${ }^{e}$ siècle et du début du XIX siècle sous l'appellation d' "école anglaise ", "école française " et " école latine ".

L'école anglaise a été mise en place en 1789 à la suite de plaintes des pères qui trouvaient le Collège trop exclusivement consacré à la formation de la relève cléricale ${ }^{27}$. Un prospectus de 1826 indique clairement de quoi il est question. On y enseigne les matières suivantes : «lecture, écriture, orthographe, les règles de commerce, la tenue de livres "; il s'agit donc de ce qui sera appelé plus tard un " cours commercial". L'école française quant à elle, selon le même document, propose ce programme : "lecture en latin et en français, écriture, calcul, premiers éléments de la langue française ». Cette formation vient, pour certains élèves, prolonger l'apprentissage reçu d'un précepteur, chez un curé ou dans une petite école et, pour d'autres, en tenir lieu, ce qui permettait à certains parents de faire bénéficier leur fils d'une alphabétisation payante, protégée de la fréquentation des enfants du peuple massés dans les " petites écoles " gratuites que les sulpiciens tenaient juste en face du Collège ${ }^{28}$. Ce programme deviendra plus tard "préparatoire » au cours classique, mais durant la période étudiée, seule une minorité de ceux qui fréquentaient l'école française entrait effectivement par la suite à l'école latine.

L'historiographie souligne abondamment combien était, selon les perspectives, sélectif ou élitiste ce long cours de latin. Le taux de persévérance est de fait extrêmement bas, ce qui est fort banal. Il ne s'agit pas d'un trait propre au Collège de Montréal, ni au Canada, ni même au XIX ${ }^{\mathrm{e}}$ siècle $^{29}$. Les études prolongées coûtent cher et la formation qu'elles donnent n'est nécessaire que pour accéder à un nombre restreint de positions. C'est ce qui explique ces histogrammes dans lesquels les sections sont, vers le haut, de plus en plus étroites. J'ai pu ainsi estimer qu'au début du $\mathrm{XIX}^{\mathrm{e}}$ siècle, moins de $5 \%$ des inscrits en première année de latin se rendaient au bout du parcours de huit années. Évoquer le manque de courage ou de facultés des enfants ou de moyens financiers des parents ne rend pas compte de la division en cycles que révèle l'analyse des effectifs par classes du cours latin (Graphique 9).

Il existe par exemple une demande manifeste pour une formation latine courte (une ou deux années, ce qu'on appelle le " cours de grammaire »), ce qui permet de mieux borner le phénomène de l'effilochement au fil des années du cursus prévu. En effet, il apparaît nettement que les deux premières années constituent en elles-mêmes un cycle particulier à l'intérieur du parcours latin de huit années. On ne peut donc considérer que les enfants qui ne vont pas plus loin que la syntaxe " abandonnent" un cours d'étude auquel ils n'ont dans les faits sans doute jamais prétendu. Si l'on se place dans une perspective d'évaluation des programmes, on pourrait tout aussi bien dire qu'ils " réussissent " le cours de grammaire.

On peut se faire une meilleure idée de la nature de l'enseignement dispensé en dirigeant son attention vers l'âge des collégiens fréquentant chaque formation ou cycle identifié. Si l'éventail paraît considérable, se dessine malgré tout une tendance à la distribution des élèves en classe d'âge. En 1826 par exemple, l'école anglaise s'adresse 

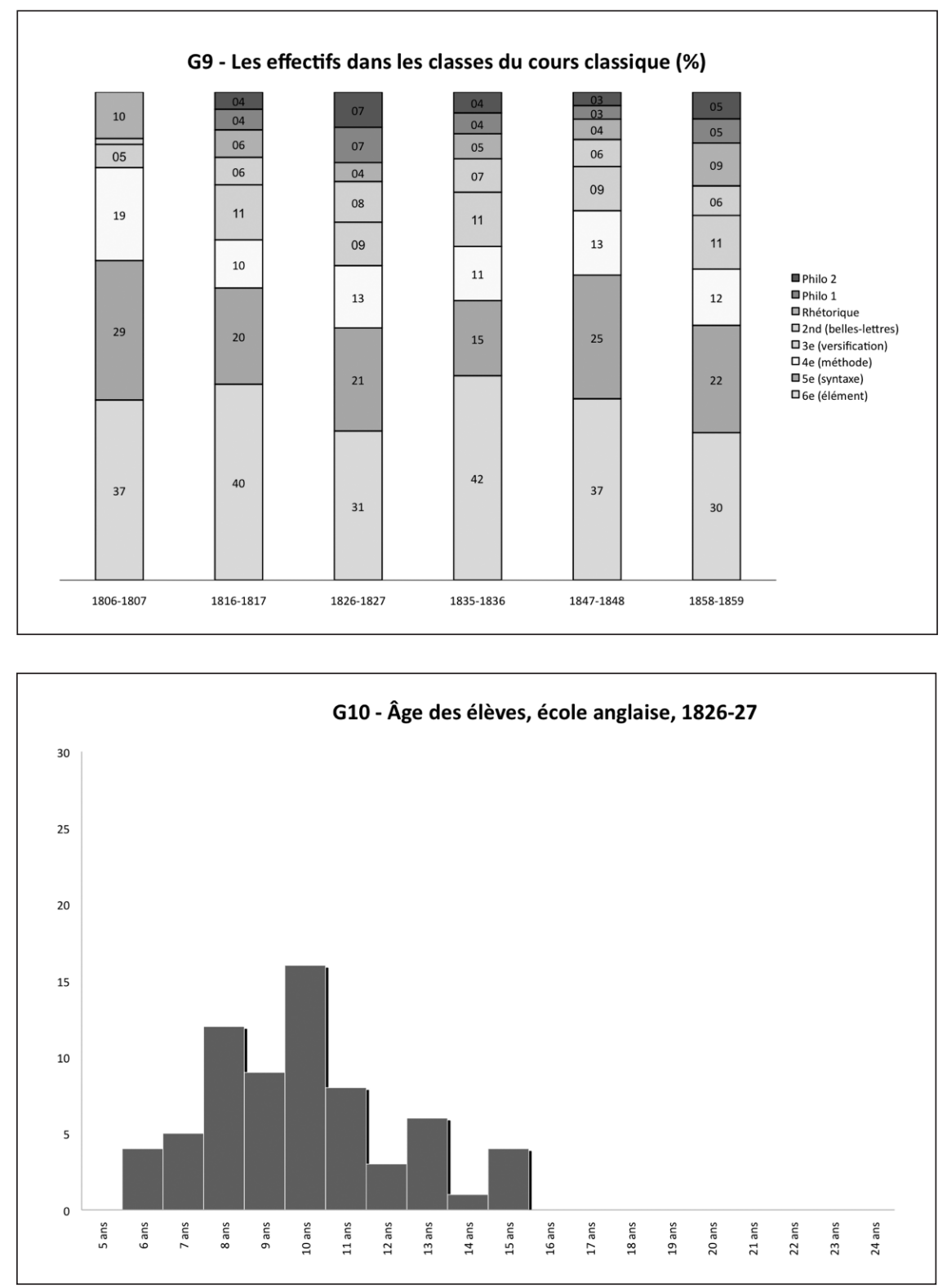

particulièrement à des garçons jeunes, de 8 à 11 ans, tandis que l'école française accueille des enfants plus vieux, de 10 à 13 ans (graphiques 10 et 11).

Ceux qui suivaient les deux premières années du cours latin ont généralement entre 11 et 15 ans (ici, un âge modal, 12 ans, se dégage nettement), alors que ceux qui fréquentent le second cycle ${ }^{30}$, beaucoup moins nombreux, ont entre 13 et 21 ans (graphiques 12 et 13). 

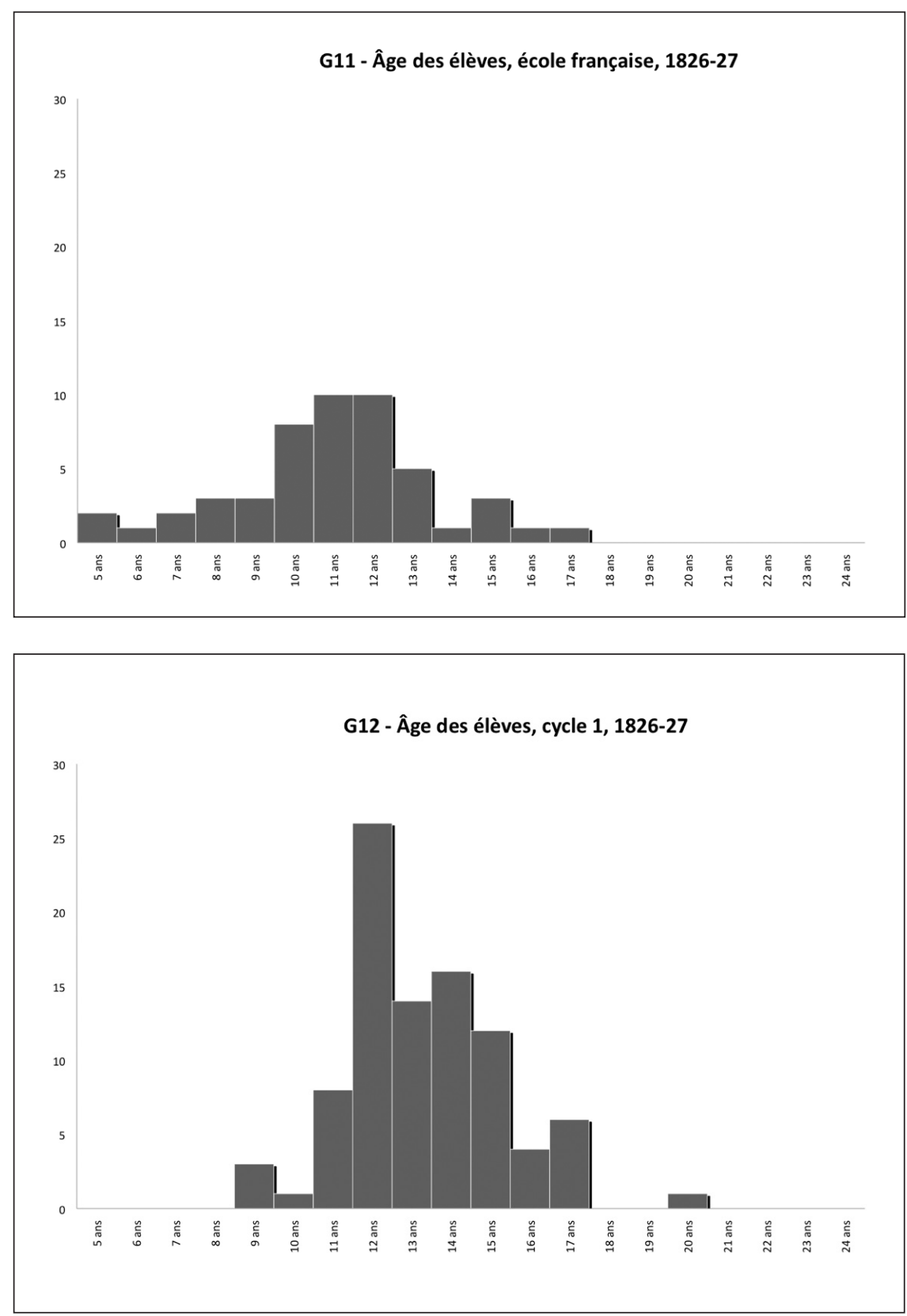

Une telle présentation accrédite l'image d'une institution " totale " à l'intérieur des murs de laquelle des générations grandissent, passant de classe en classe et de cycle en cycle selon une progression pédagogique linéaire. C'est pourtant une représentation globalement fausse. 

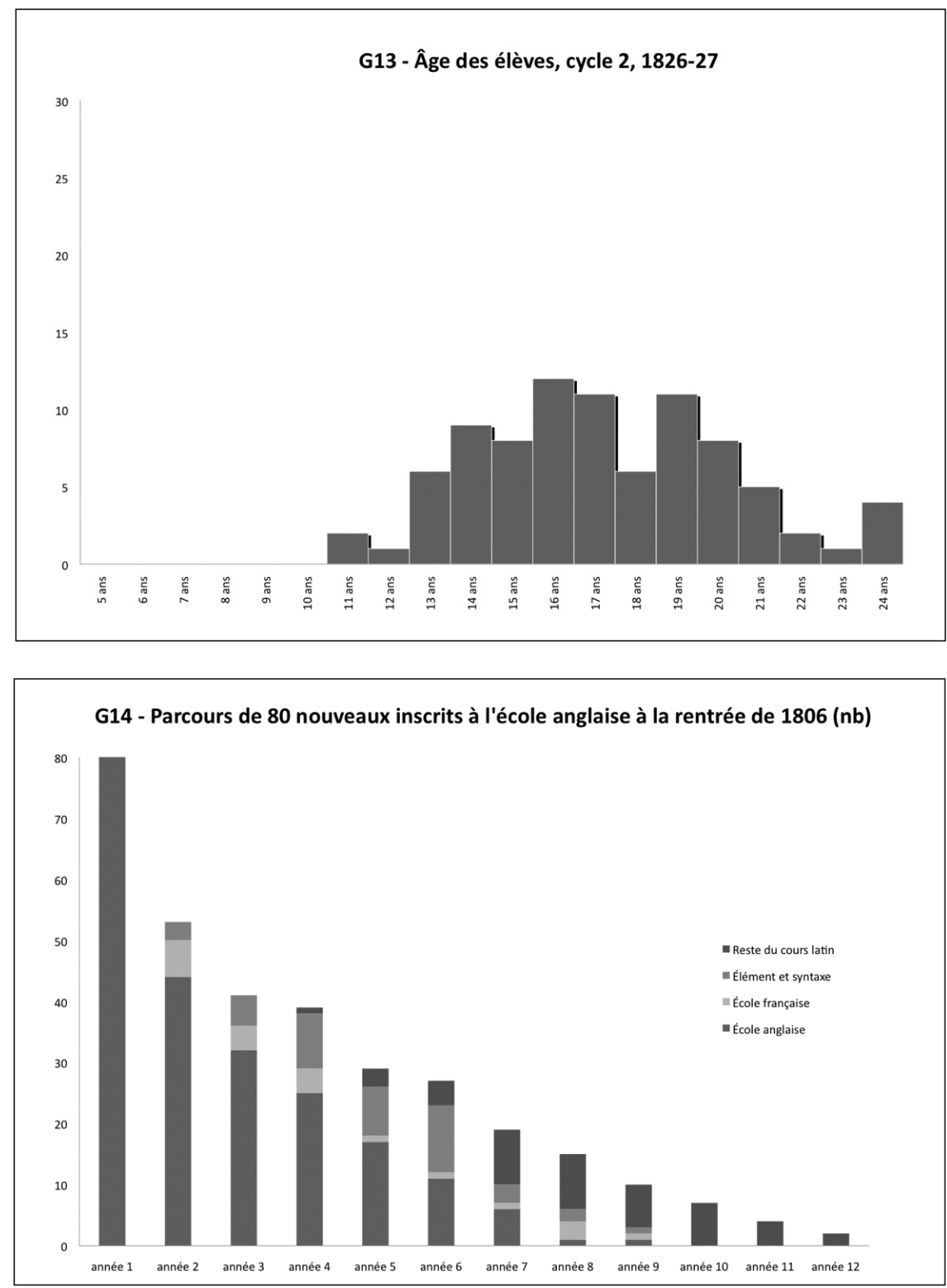

J'ai analysé le destin au fil des années et au sein de l'établissement des petits nouveaux inscrits à la rentrée de 1806. Ils sont cette année-là environ 150 .

- La moitié d'entre eux se dirigent ou sont dirigés vers l'école anglaise (Graphique 14).

S'agit-il d'une première étape qui les conduira vers le cours français puis vers le 
latin? Pour le tiers d'entre eux, l'expérience au Collège se résumera de toute manière à une seule année, passée à l'école anglaise. Peu, $12 \%$, intégreront l'école française, mais le quart d'entre eux finiront par faire du latin. Certains connaîtront même une carrière remarquablement longue à l'école anglaise - ce qui pose question quant à la formule pédagogique employée — , telle celle de William Eidam qui sera pendant huit ans inscrit à ce programme. Au total, l'école anglaise est une formation en ellemême pour la plupart de ceux qui la suivent (ce cours commercial élémentaire sera l'horizon indépassable d'environ $70 \%$ d'entre eux), et non la première marche qui conduit aux études latines.

- Quarante-six des 150 nouveaux de 1806 sont placés à l'école française, qui sera plus tard appelée « cours préparatoire " (Graphique 15).

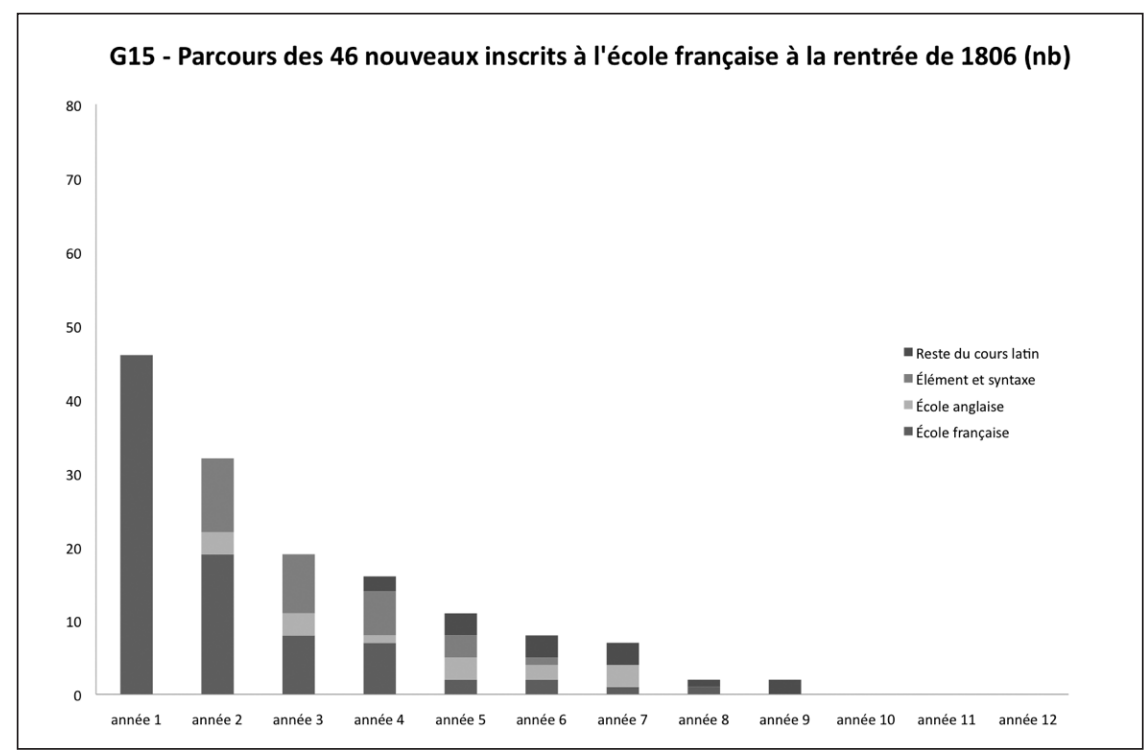

Cette formation remplit plusieurs rôles. Pour certains, elle est une école primaire privée, qui permet l'alphabétisation à l'abri des fils du peuple qui fréquentent les écoles charitables. Elle instruit aussi des enfants plus vieux qui viennent y prolonger une instruction commencée ailleurs. Enfin, l'école française accueille des élèves que les parents souhaitent scolariser, mais que les prêtres jugent inaptes au latin ${ }^{31}$. Tel est par exemple le cas du jeune Basile Plessis-Bélair qui sortira du Collège à 16 ans après avoir passé huit années consécutives à tracer des lettres dans son abécédaire et à apprendre le catéchisme sur les bancs de l'école française ${ }^{32}$. Au total, celle-ci est une formation en elle-même pour plus de la moitié des enfants qui la fréquentent; ceux qui la quittent vont soit vers l'anglaise $(35 \%)$ soit vers le latin $(65 \%)$.

- Enfin, 26 petits nouveaux de 1806 entrent directement en élément latin (Graphique 16). 


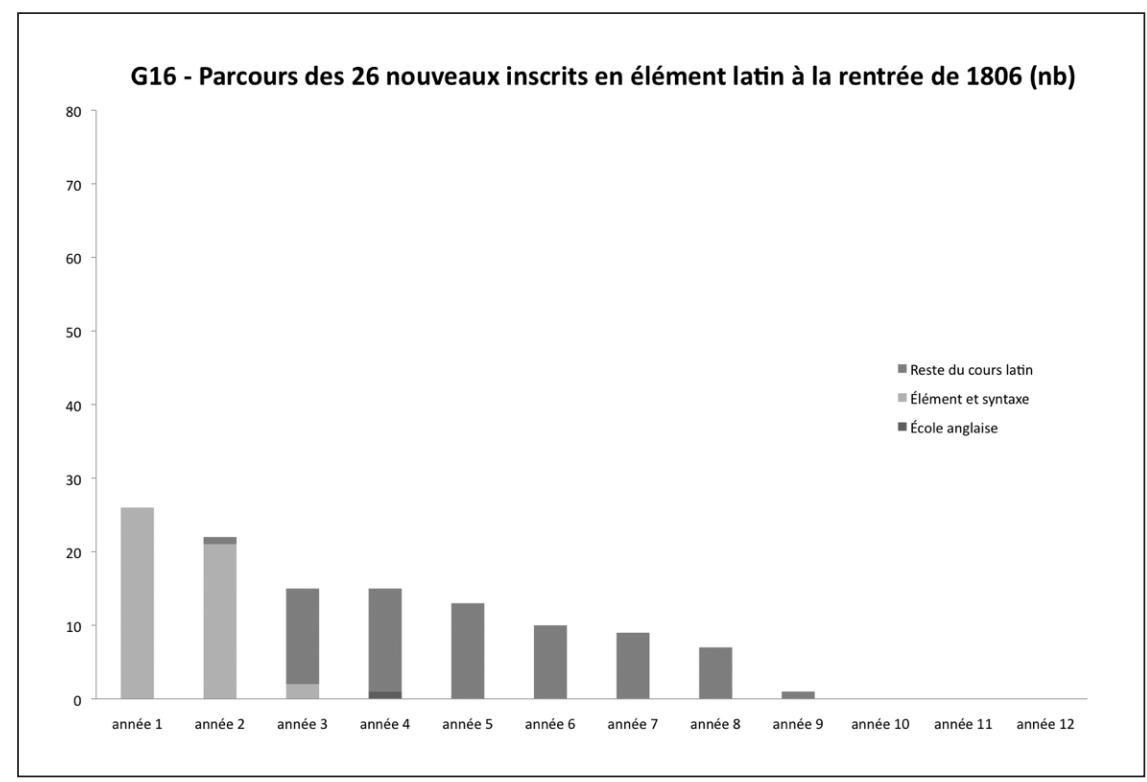

Presque tous suivent en $5^{\mathrm{e}}$ (syntaxe), mais, selon la logique de cycles évoquée plus haut, une partie importante du groupe ne poursuit pas au-delà de cette étape. La moitié du noyau de départ entame la formation longue en 1808. Par la suite, chaque année, environ deux d'entre eux quittent le Collège de sorte que ce sont sept des 26 inscrits en 1806 qui finiront le cours complet de huit années ${ }^{33}$.

Lorsque l'on considère le collège comme un lieu d'éducation aux filières diverses quoiqu'interconnectées - école anglaise, école française, latin cycle court, second cycle du cours latin — , et non en l'évaluant à partir d'une norme qui ne se mettra en place que plus tard, il n'apparaît pas comme une institution élitiste et élitaire, mais se présente comme une maison ouverte aux besoins multiples du milieu, c'est-à-dire offrant surtout des études pratiques et courtes. La figure de l'adolescent pensionnaire absorbé par les thèmes et les versions et plongé tout entier dans le monde antique s'estompe devant celle de l'enfant occupé à apprendre l'arithmétique, les langues vivantes et les bases de la culture humaniste. Au début du siècle, ce sont plus de $80 \%$ des collégiens qui sont engagés dans un tel programme. À la fin des années 1860, ils formeront encore environ $60 \%$ des effectifs.

Dans le même temps, un processus est en marche, qui tend vers la rationalisation et l'homogénéisation de la formation. L'affirmation progressive des études latines longues est une évolution qui configure fortement l'histoire du Collège, et peut-être du système secondaire québécois en général. Le graphique 17 exprime à la fois cette pluralité initiale des enseignements collégiaux et leur dépolarisation dans le temps. Au début du XIX e siècle, les cours sans latin attirent plus de collégiens que le cours latin. Cependant, dès les années 1810, ce dernier fait jeu égal avec les autres filières. Le creux dans les courbes enregistré au milieu des années 1830 est en fait imputable à un double phénomène : la disparition de l'école anglaise, qui intervient probablement 


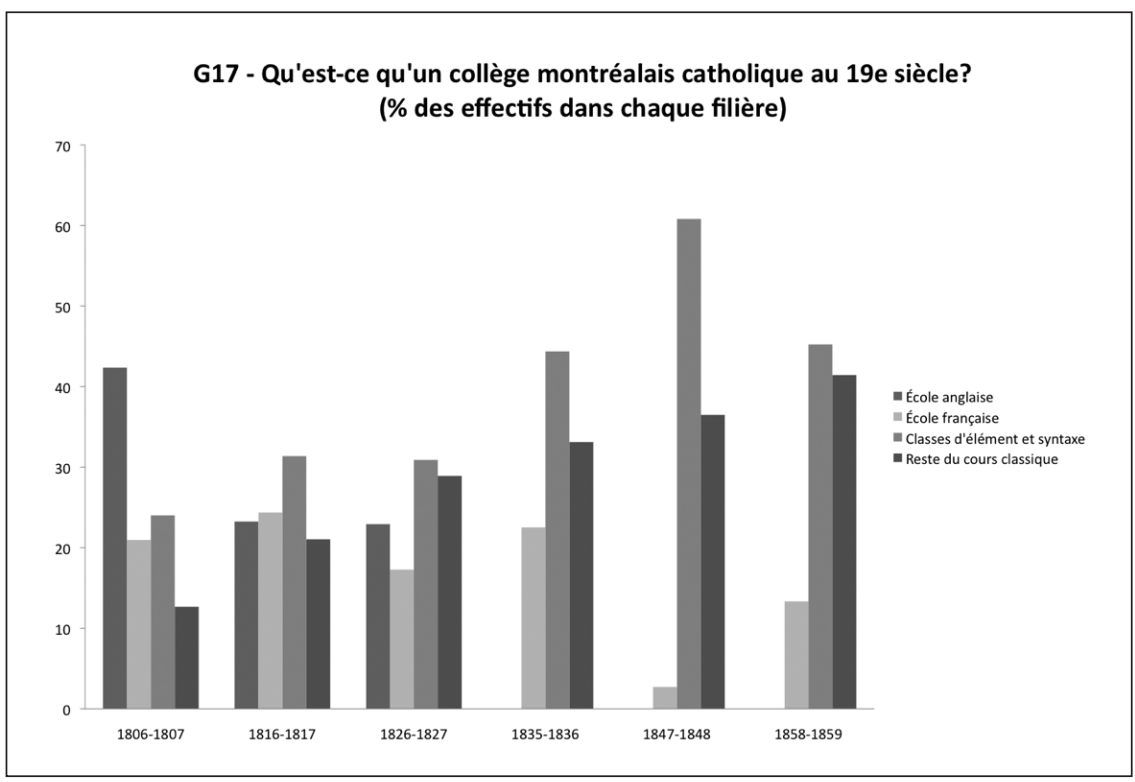

en 1830 ou 1831, et le tassement des inscriptions au cours latin, qui est lié à la crise politique et à l'augmentation du prix de la pension ${ }^{34}$. La reprise des années 1840 constitue le triomphe des humanités, et plus précisément du premier cycle qui accueille $60 \%$ des inscrits. Le Collège de Montréal offre alors, sans doute aux fils de commerçants et surtout d'artisans montréalais dont les parents développent un certain goût de la distinction, une éducation classique courte. La décroissance des années 1850 ne doit pas être comprise comme une désaffection, mais comme le produit d'une concurrence vive entre les divers établissements qui cherchent désormais à séduire cette nouvelle clientèle. Parallèlement, l'intérêt pour les études latines longues ne cesse de croître, ce qui signale l'accroissement des effectifs dans les grandes classes du cours latin qui regroupent en 1858-59 environ $40 \%$ des collégiens. Ce phénomène se répercute sur l'âge moyen des élèves (Graphique 18). Jusque dans les années 1840, le Collège accueille autant d'enfants entre 7 et 12 ans que de ce que nous appellerions des adolescents ou de jeunes adultes de 13 à 20 ans. Les sources en rendent très bien compte, parlant constamment des " petits " qu'il faut séparer et protéger des «grands ». À partir du milieu du XIXe siècle, le Collège de Montréal n'est plus le lieu de l'enfance.

Par ailleurs, l'organisation en classe d'âge devient sans doute à ce moment de plus en plus manifeste. Une recherche reste à conduire à cet effet, mais si l'hypothèse devait se confirmer, elle serait à porter au crédit de la thèse de l'embourgeoisement. En effet, l'hétérogénéité des âges dans chaque niveau témoigne d'une ouverture de l'institution à la diversité des itinéraires possibles : école primaire débutée tardivement, interruption d'études, stagnation à un stade particulier du cursus ou changements d'orientation. La rigidification de l'offre collégiale (un cours latin long dans lequel à chaque classe correspond un âge) rend le système moins tolérant à l'égard des 


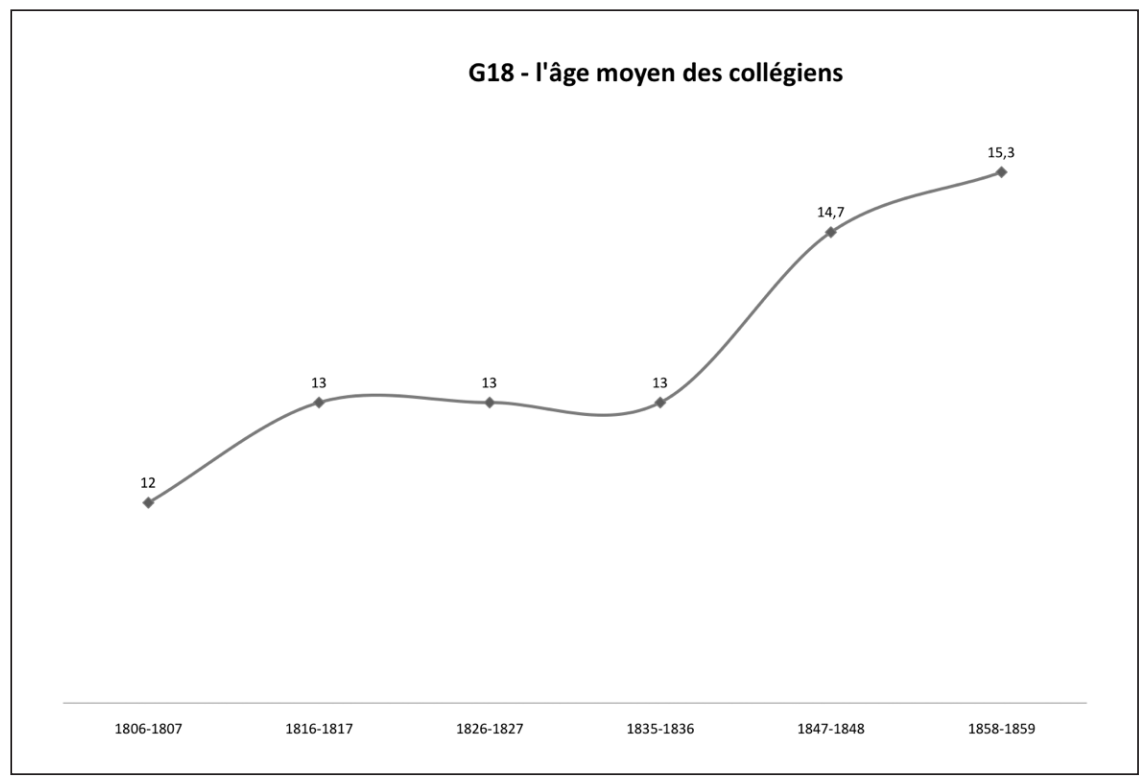

parcours atypiques qui sont plus souvent le fait d'enfants issus de milieux modestes que celui des fils de notables.

\section{E-Pour une histoire de tous les collégiens}

Dans une perspective d'histoire de l'éducation, qui plus est dans le moment intense des années de la démocratisation scolaire des années 1960, il est compréhensible que l'attention des spécialistes se soit portée vers la mesure de la fréquentation du cours secondaire complet. En effet, le problème qui se posait était celui de l'adaptation d'une pédagogie conçue comme particularisante aux catégories nouvelles qu'il s'agissait d'intégrer. Par ailleurs, le diplôme étant au XXe siècle le principal outil de mesure de la réussite, il était sans doute aussi inévitable que l'accent soit mis sur la fin plutôt que sur le parcours. Le concept de " décrochage " illustre bien cette perception : ne pas faire le cursus au complet, c'est presque comme si on n'avait rien fait. Une autre tradition incitait à ne voir dans le collégien que l'unique figure du " finissant ». En effet, les associations d'anciens, qui construisirent largement la mémoire des collèges et jusqu'à un certain point leur histoire, s'inscrivaient dans une logique de clubs dont les non-finissants étaient exclus. L'histoire culturelle elle-même, longtemps étude des formes de la culture bourgeoise, eut tendance à privilégier les matières enseignées dans les dernières années du cours classique, considérées peut-être comme le plus digne d'intérêt, au détriment des savoirs transmis dans les plus petites classes. L'histoire de l'éducation de son côté a concentré son attention vers les écoles primaires, perçues comme le berceau de l'enseignement démocratique. Quant à l'histoire politique, ou religieuse, définie comme examen de ceux qui occupent les positions de pouvoir, elle multiplia les narrations biographiques concernant des individus qui avaient presque 
toujours connu des parcours scolaires " complets ». Enfin, les maisons d'éducation elles-mêmes n'enregistrèrent que relativement peu de traces du passage des collégiens qui ne correspondaient pas au modèle du " finissant ", car ceux qui avaient le pouvoir dans les établissements étaient les professeurs de Belles-lettres, de Rhétorique et de Philosophie. Ils dédaignaient ou ignoraient les populeuses petites classes.

C'est par la conjonction de ces déterminismes historiques et historiographiques que la vaste majorité des élèves a disparu du radar. L'histoire des collèges classiques a été construite dans la perspective des vainqueurs (et rédigée par eux), ou du moins de ceux qui se pensèrent ou furent pensés comme tels : " anciens ", "finissants ", " diplômés ». De sorte qu'une des marques de leur triomphe aura justement été de faire plonger dans l'oubli le parcours de la plupart des enfants qui partagèrent avec eux les bancs des petites classes. Pourtant, lorsque l'on considère une période durant laquelle il n'existe pas de diplôme et de rigidité dans l'itinéraire, où les maisons d'éducation ont des vocations plurielles, il semble essentiel de repartir à la recherche du cheminement vécu par tous les collégiens. L'attention doit être déplacée de l'aristocratie des rhétoriciens et des philosophes vers la masse de ceux qui se contentèrent du cours de grammaire, ou ne firent jamais de latin. Au Collège de Montréal, au XIX siècle, ils constituèrent probablement autour de $90 \%$ des effectifs. Qui étaient-ils, qu'ont-ils appris dans les salles de classe et plus généralement quelle expérience le collège a-t-il représentée pour eux? Certains ont pu y passer moins d'un an et d'autres plus de dix. D'autres firent tous les cours, anglais, français, latin. D'autres encore n'intégrèrent l'établissement que tard, pour faire leur rhétorique. Beaucoup ne connurent que les petites classes latines. Le collège n'est pas, ou pas seulement, le lieu de dressage des groupes dominants et d'acquisition de l'habitus bourgeois. Il apparaît plutôt sous la forme d'un espace de vie complexe dans lequel plusieurs mondes se croisent et de multiples destins peuvent se forger. Pour le comprendre, il faut tenir compte de l'ensemble des enfants et non des seuls "finissants ", un terme que du reste on ne lit pas dans les archives du XIX ${ }^{\mathrm{e}}$ siècle $^{35}$.

Contre une tradition sociologique, inspirée par Émile Durkheim ${ }^{36}$, une histoire sociale de la formation collégiale ne devrait par conséquent pas se contenter de supposer que la fonction unique de ces institutions était la production d'un habitus bourgeois par le pensionnat et le latin. L'expérience du collège n'est pas seulement celle de l'exil à l'égard de la chaleur, éventuelle, du foyer. Elle n'est pas, du moins pas pour tous, celle de prison, mais au contraire d'un espace de porosité : un territoire intéressant justement, fascinant, parce qu'il dépasse le paradigme foucaldien du contrôle panoptique. Le dispositif disciplinaire collégial est ouvert, mais poursuit sans relâche un idéal de fermeture. La discipline apparaît alors comme l'effort pour contenir l'astuce qui cherche à ruser avec elle.

À l'évidence, les itinéraires étaient très variés et il convient de qualifier cette variété, d'y déceler des régularités, de comprendre ce qu'elles signifiaient concrètement dans le quotidien des enfants, de ne pas faire comme si une, deux, quatre années d'éducation, aussi divergentes soient-elles par rapport au cursus idéal de la norme élitaire minoritaire, étaient assimilables à un parcours en école paroissiale ou à une absence totale de scolarisation. Il se trouve que l'expérience collégiale la plus commune 
était celle d'un passage à durée variable. C'est pourquoi ma recherche s'orientera pour le futur vers une lecture de l'établissement comme un espace de formation pluriel et non comme l'incarnation du moule idéaltypique, théorique, trop généralement proposé par l'historiographique. En particulier, les notions d'écoles, de filières, de cycles, de classes doivent primer et supplanter l'idée de cours ou de collège " classique ", au moins dans l'espace-temps envisagé. À cette complexité devra être ajoutée celle qu'offrirait la prolongation de cette série dans le temps et la mise en comparaison des résultats obtenus pour le cas montréalais avec ceux des autres institutions « secondaires ", chacune possédant au Québec sa propre histoire, tissée d'adaptations successives et multiples, de sorte qu'aucune situation empirique ne puisse être assimilée a priori aux modèles théoriques que l'histoire de l'éducation propose. À terme, on s'autorise alors à penser la construction d'un ensemble d'habitudes culturelles, qui sont peutêtre aussi sociales et politiques et ne relèveraient ni du monde de l'analphabétisme ou du déchiffrage, ni de plain-pied de celui de la culture reconnue comme savante, mais d'un entre-deux qu'il reste à décrire ${ }^{37}$. Ce qui est ici cerné, c'est la condition de possibilité d'habitus qui définiront des groupes intermédiaires ${ }^{38}$, qui, par le collège, accèdent à un type de connaissance semi-éclairée dépassant de beaucoup le simple apprentissage du savoir lire, écrire et compter des petites écoles paroissiales, sans pour autant atteindre le niveau du second cycle du cours classique. Entre la littéracie fruste et le maniement distingué de l'humanisme existe un espace culturel et sociologique qui ne se constitue pas en dehors de la culture savante, mais à l'écart de ses formes les plus approfondies, comme en ses marges. L'hypothèse sur laquelle s'achève ce travail est celle d'une urbanité parcourue par le désir d'une instruction poussée au-delà des rudiments, mais qui peut s'incarner en une variété d'itinéraires, de degrés et de contenus. Cette panoplie doit rendre compte à la fois de stratégies différenciées de promotion et d'une certaine complexité des marqueurs qui régulent la vie sociale en fonction du degré d'initiation au monde lettré.

\section{Conclusion}

Revenons pour finir à cette idée : infime était la proportion des élèves du premier $\mathrm{XIX}^{\mathrm{e}}$ siècle qui vécut la théorie proposée en modèle par l'historiographie : celui du pensionnaire passant toute son enfance et son adolescence prisonnier derrière les murs concentrationnaires d'un établissement dans lequel on le gavera de latin. Dans les faits, beaucoup de collégiens montréalais ne faisaient même pas de latin, nombre d'entre eux étaient externes. On entrait au collège et on en sortait à des âges très différents, on le fréquentait pour des périodes variables en y suivant des parcours divers. Autrement dit, la différence paraît immense entre certaines représentations historiographiques et mémorielles et celle livrée par l'analyse quantitative. Selon une de ces logiques contre-intuitives qui réjouissent toujours les historiens, il apparait que le mouvement de l'histoire des collèges — mais il faudrait pour mieux le poser élargir le terrain de l'enquête — ne conduit pas de l'élitisme à la démocratisation, mais, avant celle-ci, de l'ouverture à l'homogénéisation. Une histoire sociale du collégial se dessine donc, valable au moins pour une certaine catégorie d'établissements, qui part 
de l'externat et de familles désireuses de formations pratiques et courtes vers un internat pour adolescents engagés dans un programme de plus en plus théorique et long. Cette évolution est liée à la mise en place d'un réseau d'institutions « secondaires » qui permet une spécialisation (relative ${ }^{39}$ ) de chaque maison en fonction de divers groupes sociaux, qu'elles contribuent par la même à construire. Dans ce contexte, les collèges dirigés par les prêtres, les plus anciens, tendent à se spécialiser dans le pensionnat classique, dispositif qui acquiert peu à peu un prestige couronnant celui qui en est issu d'une légitimité évidente.

\section{Notes}

1 Cette recherche a été rendue possible grâce au soutien du Fonds québécois de la recherche sur la société et la culture et du Conseil de recherches en sciences humaines du Canada. Je remercie mes collègues Christine Hudon et Louise Bienvenue, ainsi que les évaluateurs anonymes pour leurs commentaires et les étudiants qui m'ont secondé; en particulier : Éveline Bousquet, Guillaume Fortier, Maxime RaymondDufour et Émilie Tanniou. Je remercie encore les prêtres de Saint-Sulpice et leur équipe d'archivistes : Caroline, David et Marc Lacasse.

2 Il faudra de plus en préciser l'importance relative.

3 Sur le sens du "discours ", chez Foucault, voir Paul Veyne, Foucault, sa pensée, sa personne, Paris, Albin Michel, 2008, 46.

4 On peut raisonnablement imaginer qu'il surgit à la confluence de la mémoire des " anciens ", qui aiment à se penser en vainqueurs, des stratégies publicitaires des institutions elles-mêmes, qui misent sur une image élitiste, et des attaques de leurs détracteurs qui condamnent cet élitisme supposé; toutes choses qui appartiennent à l'ordre des représentations, de leur production et de leurs usages.

5 Roger Chartier, Marie-Madeleine Compère et Dominique Julia, L'éducation en France du XVIe au XVIII siècle, Paris, SEDES, 1976, chapitre 5.

6 Par exemple : Pierre Bourdieu et Jean-Claude Passeron, Les héritiers, Paris, Éditions de Minuit, 1964 ; Antoine Prost, Histoire de l'enseignement en France, 1800-1967, Paris, Armand Colin, 1968 ; Alain Plessis, "Une France bourgeoise » dans Histoire de la France, Les formes de la culture, Paris, Seuil, 1993, 272-278. Au Québec, le même défi se pose. Dans les années 1960, la commission Parent signale l'allure non démocratique des effectifs collégiaux — une " mince couche de population, généralement privilégiée à tous points de vue » — comme l'un des principaux problèmes qui doivent justifier la refonte du système éducatif (Rapport de la Commission royale d'enquête sur l'enseignement dans la province de Québec, 2, 1964, chapitre 1). Par ailleurs, un ensemble de textes à caractère historique permettent de forger la représentation du collège comme institution fondamentalement bourgeoise. Les histoires de collèges alimentent depuis longtemps l'idée de distinction sociale. En 1954, les porte-parole de la fédération des collèges classiques écrivent eux-mêmes que leurs établissements sont réservés à une élite sociale (Claude Corbo, Les Jésuites québécois et le cours classique après 1945, Québec, Éditions du Septentrion, 2004, 66). Finalement, l'histoire sociale accrédite dans l'ensemble l'image du collège classique bourgeois (par exemple John A. Dickinson et Brian Young, Brève histoire socio-économique du Québec, Septentrion, Québec, 1995, 109-110)

7 J'observe cependant que les études historiques qui considèrent en détail la population scolaire d'une maison particulière à une époque particulière font très souvent la démonstration de sa mixité sociale et de la diversité de ses parcours. Dans leur brillante étude considérant quatre collèges français du XVIII siècle, Willem Frijhoff et Dominique Julia les trouvent plus démocratiques que ceux du XIX et du premier $\mathrm{XX}^{\mathrm{e}}$ 
siècle (École et société dans la France d'Ancien Régime. Quatre exemples: Auch, Avallon, Condom et Gisors, Paris, Armand Colin, 1975). Félix Bouvier observe pour le Québec du XXe siècle le même phénomène, qu'il attribue au contexte de la "région neuve " qu'il étudie (Histoire du Séminaire de Mont-Laurier. Formation d'une élite et d'une classe moyenne, Montréal, Fides, 2005, 223). Ces constantes hésitations quant au degré d'ouverture des maisons d'éducation secondaire se retrouvent encore dans l'ouvrage collectif publié à l'occasion du bicentenaire du Lycée français (Pierre Caspard, « Du passé, faisons table ronde » dans Pierre Caspard, Jean-Noël Luc, Philippe Savoie (dir.), Lycées, lycéens, lycéennes. Deux siècles d'histoire, Paris, Institut National de Recherche Pédagogique, 2005, 484). La conclusion s'impose d'elle-même : nous en savons trop peu sur les clientèles des collèges en particulier pour nous faire une idée de leur évolution d'ensemble.

8 Claude Galarneau, Les collèges classiques au Canada français, Montréal, Fides, 1978. Le chapitre 6 utilise une série d'études, pour la plupart réalisées à partir des listes d'élèves publiées par les collèges. Ces documents sont pourtant problématiques, car ils confondent les formations et sont d'une fiabilité douteuse pour les périodes anciennes. Dans l'ensemble, le texte croise des données hétérogènes et ponctuelles sensées rendre compte des évolutions sur une très longue période, de sorte que le bilan plonge le lecteur dans une grande confusion.

9 Claude Galarneau, Les collèges..., 142.

10 APSSM. On trouve les volumes et cahiers dans le fonds "Collège de Montréal », Boîtes I2: 8 à 12 .

11 Il est possible d'obtenir ces informations pour un nombre significatif de cas, en faisant usage des registres de baptême principalement. On marcherait alors les traces des anciennes enquêtes d'histoire sociale et quantitative des populations scolaires initiées pour le monde francophone dès les années 1950 par François de Dainville («Effectifs des collèges et scolarité aux XVII et XVIII ${ }^{e}$ siècles dans le nord-est de la France ", Population, 10, 3 (juillet-septembre 1955), 455-488 et " Collèges et fréquentation scolaire au XVII' siècle ", Population, 12, 3 (juillet-septembre 1957), 467-494). Voir aussi Jean de Viguerie, «Les origines sociales et géographiques des élèves du collège de Lectoure dans la seconde moitié du XVIII ${ }^{e}$ siècle » dans Actes du $95^{\circ}$ congrès national des sociétés savantes, Paris, Bibliothèque nationale, 1974. Pour le Québec: Ulric Lévesques, "Les élèves du collège de Sainte-Anne-de-la-Pocatière, 1829-1842 ", Revue d'histoire de l'Amérique française, 21, 4 (mars 1968), 774-791) ; Jean-Paul Simard et Bérard Riverin, "Origine géographique et sociale des étudiants du Petit Séminaire de Chicoutimi et leur orientation socioprofessionnelle : 1873-1930", Sessions d'étude, Société canadienne d'histoire de l'Église catholique, 1973, 33-53; Claude Lessard, "Les élèves des séminaires de Nicolet et de Trois-Rivières : leur origine géographique, leur persévérance ", Revue d'ethnologie du Québec, 2 (1975), 57-76; Claude Lessard, "Les élèves des séminaires de Nicolet : leur origine sociale, leur persévérance 1803-1969 ", Revue d'ethnologie du Québec, 3 (1976), 29-50 ; Claude Lessard, «L'origine sociale des élèves du séminaire de Trois-Rivières (1860-1976) ", Revue d'ethnologie du Québec, 10 (1979), 57-70; Félix Bouvier, Histoire du Séminaire de Mont-Laurier..., chapitres 8 à 10. Les plus anciens travaux dirigent leur attention sur le critère socioprofessionnel, car l'intention est d'illustrer l'inégalité sociale fondamentale d'un système qu'il s'agit de réformer. C'est pourquoi l'attention fine aux possibles variations de la forme des clientèles dans le temps y est ordinairement insuffisante, tout comme celle portée à d'autres critères tout aussi fondamentaux. La notion de cheminement, en particulier, échappe à peu près.

12 Marie-Madeleine Compère, Du collège au lycée (1500-1850), Paris, Gallimard-Julliard, 1985, 159.

13 Jean-Claude Caron, «Les jeunes à l'école : collégiens et lycéens en France et en Europe (fin XVIII--fin XIX siècle) ", dans Giovanni Levi et Jean-Claude Schmitt, dir., Histoire des jeunes en Occident, 2, Paris, Seuil, 1996. 
14 Marie-Madeleine Compère, Du collège au lycée, 131.

15 Claude Galarneau, Les collèges..., 141.

16 Claude Galarneau avait pu sur ce point être plus précis (Les collèges..., 131-134); voir aussi Claude Lessard, "Les élèves des séminaires... »; Jean-Paul Simard et Bérard Riverin, "Origine géographique et sociale...».

17 L'origine des " Européens " est tenue pour non significative, l'économe inscrivant manifestement des Montréalais sous cette classe.

18 Claude Galarneau, Les collèges..., 134

19 APSSM, P1:11.3-100 «Deux prospectus du Collège de Montréal en langue anglaise, celui de 1838 est tiré de The Metropolitain Catholic Almanac, de Baltimore ", $1838-1840$.

20 Pour Nicolet, Claude Lessard attribue cette présence américaine significative au fait que la Nouvelle-Angleterre accueillait de nombreux prêtres anciens de ce petit séminaire vers lequel ils dirigent des enfants de leur paroisse, ainsi qu'au manque d'institutions scolaires catholiques aux États-Unis. Il semble qu'il s'agisse, pour la période qui nous intéresse, de collégiens d'ascendance irlandaise et non de Franco-Américains. (Claude Lessard, "Les élèves des séminaires... », 64-65). Au XIX” siècle, le Collège Sainte-Marie de Montréal est lui aussi fréquenté par une population mixte sur le plan linguistique. Comme au Collège de Montréal, les anglophones sont des citadins ou des Américains. Le cours commercial s'y donne en anglais et, à partir de 1889, il existe un cours classique anglais (Jean Cinq-Mars, Histoire du Collège Sainte-Marie...).

21 Une remarquable étude existe pour le cas suisse : Pierre Caspard, "Les changes linguistiques d'adolescents. Une pratique éducative, $\mathrm{XVII}^{\mathrm{e}}-\mathrm{XIX}{ }^{\mathrm{e}}$ siècles ", Revue historique neuchâteloise (janvier - juin 2000), 82 p.

22 Marie-Madeleine Compère et Philippe Savoie : "L'établissement secondaire et l'histoire de l'éducation ", Histoire de l'éducation, 90, mai 2001, 5-20.

23 Jean Cinq-Mars (Histoire du Collège Sainte-Marie de Montréal, 1848-1969, Hurtubise $\mathrm{HMH}, 1978,58-59)$ identifie bien cette réalité. En revanche, elle passe inaperçue dans Olivier Maurault et Antonio Dansereau, Le Collège de Montréal, 1767-1967, Montréal, 1967, 574 p.

24 Ulric Lévesques, constatant le phénomène à la Pocatière, propose qu’il génère inévitablement des "problèmes d'adaptation ». Il juge du reste globalement que le Collège fonctionne dans la première moitié du XIX ${ }^{e}$ siècle de manière irrégulière, ce qui rend difficile son analyse (Ulric Lévesques, "Les élèves du collège de Sainte-Anne-de-laPocatière... ", 780).

25 Par exemple Yvan Lamonde, La philosophie et son enseignement au Québec (1665-1920), Montréal, Hurtubise-HMH, 1980 ; Marc-André Bernier, «Portrait de l'éloquence à Québec (1760-1840) ", dans Bernard Andrès et Marc-André Bernier, dir., Portrait des arts, des lettres et de l'éloquence au Québec (1760-1840), Québec, Les Presses de l'Université Laval, 2002.

26 Les plus anciens travaux dirigent leur attention sur le critère socioprofessionnel, car l'intention est d'illustrer l'inégalité sociale fondamentale d'un système qu'il s'agit de réformer. C'est pourquoi l'attention fine aux possibles variations de la forme des clientèles dans le temps y est ordinairement insuffisante, tout comme celle portée à d'autres critères tout aussi fondamentaux. La notion de cheminement, en particulier, échappe à peu près. Willem Frijhoff et Dominique Julia proposèrent en 1975 un modèle méthodologique qui permet de retrouver la logique propre des collèges (ÉEcole et société dans la France d'Ancien Régime...). Jean-Cinq Mars publie d'intéressantes statistiques pour le Collège Sainte-Marie (Histoire du Collège Sainte-Marie..., Annexes 2 et 9). C'est un grand mérite de l'étude de Félix Bouvier que d'être attentive aux questions de cheminement, de cycles et de cours (Histoire du Séminaire de MontLaurier..., 187-217). 
27 APSSM, P1 : 11.3-15, "Compte rendu des délibérations du conseil de la Fabrique de Notre-Dame de Montréal. Extraits concernant le Collège de Montréal, de 1773 à 1806 » et A.-Z. Massicotte, "Une page de l'histoire du Collège de Montréal », Bulletin de recherches historiques, 23, 7 (juillet 1917), 207-211.

28 Ollivier Hubert, «Petites écoles et collèges sulpiciens ", dans Dominique Deslandres, John Dickinson et Ollivier Hubert (dir.), Les Sulpiciens de Montréal : une histoire de pouvoir et de discrétion, 1657-2007, Saint-Laurent, Fides, 2007, 395-444.

29 François Lebrun, Marc Vénard, Jean Quéniart, Histoire de l'enseignement et de l'éducation, 2, 1480-1789, Paris, Perrin, 2003 (1981), 362-372.

30 Qu'il faudrait sans doute subdiviser lui aussi autour de l'année de versification.

31 APSSM, I2 : 8, Registres des pensionnaires de 1810-11, feuille flottante.

32 Plusieurs sources donnent les manuels utilisés à l'École française. APSSM, I2: 8 et I2 :11 « Comptes de fournitures de l'école française depuis le 2 octobre 1809 jusqu'au $1^{\text {er }}$ janvier $1810 \%$.

33 L'impression d'hécatombe doit être nuancée pour une autre raison. En effet, il faut penser que le fait de sortir d'une institution ne signifie pas nécessairement un abandon des études. En fait, l'épluchage des flux d'élèves montre que le Collège de Montréal accueille lui-même chaque année des nouveaux venus, et à tous les niveaux, ce qui signale une forte mobilité inter-établissements. Celle-ci rend hasardeuse, de plus en plus hasardeuse à mesure que passe le temps et que se multiplient les maisons d'enseignement secondaire, la mise sur pied de statistiques de fréquentation et de taux de persévérance.

34 La pension passe de $£ 14$ à $£ 18$ en 1833 et la scolarité de $£ 1$ à $£ 4$.

35 La première mention du terme dans les archives du Collège de Montréal date des années 1930.

36 Émile Durkheim, L'évolution pédagogique en France, Paris, Presses universitaires de France, 1938.

37 Une perspective qui inscrit la recherche dans une histoire sociale et politique des usages et pratiques de la culture écrite proposée notamment par Bruce Curtis, «Beyond Signature Literacy : New Research Directions ", Historical Studies in Education/Revue d'histoire de l'éducation, 19, 2 (automne 2007), 1-11.

38 Dans un article récent, Gary McCulloch lance un appel pour que l'histoire de la formation scolaire des groupes intermédiaires soit mieux étudiée, après des décennies de recherches polarisées sur l'expérience des élites sociales, des ouvriers et des paysans. Gary McCulloch, "Education and the Middle Classes: The Case of the English Grammar Schools, 1868-1944 ", History of Education, 35, 6 (novembre 2006), 689-704. Voir aussi Robert Gildea, Education in Provincial France, 1800-1914: A Study of Three Departments, Oxford, Clarendon Press, I983.

39 Combien de soi-disant « collèges classiques " sont-ils dans les faits largement des collèges commerciaux, au $\mathrm{XX}^{\mathrm{e}}$ siècle encore? 
44 Historical Studies in Education/Revue d'histoire de l'éducation

\section{Blank \\ Left \\ Page}

